\title{
GWO-LPWSN: Grey Wolf Optimization Algorithm for Node Localization Problem in Wireless Sensor Networks
}

\author{
R. Rajakumar, ${ }^{1}$ J. Amudhavel, ${ }^{2}$ P. Dhavachelvan, ${ }^{1}$ and T. Vengattaraman ${ }^{1}$ \\ ${ }^{1}$ Department of Computer Science, Pondicherry University, Puducherry, India \\ ${ }^{2}$ Department of Computer Science, KL University, Vaddeswaram, India \\ Correspondence should be addressed to R. Rajakumar; ramukshare@gmail.com
}

Received 16 November 2016; Revised 18 February 2017; Accepted 2 March 2017; Published 21 March 2017

Academic Editor: Arun K. Sangaiah

Copyright (C) 2017 R. Rajakumar et al. This is an open access article distributed under the Creative Commons Attribution License, which permits unrestricted use, distribution, and reproduction in any medium, provided the original work is properly cited.

Seyedali Mirjalili et al. (2014) introduced a completely unique metaheuristic technique particularly grey wolf optimization (GWO). This algorithm mimics the social behavior of grey wolves whereas it follows the leadership hierarchy and attacking strategy. The rising issue in wireless sensor network (WSN) is localization problem. The objective of this problem is to search out the geographical position of unknown nodes with the help of anchor nodes in WSN. In this work, GWO algorithm is incorporated to spot the correct position of unknown nodes, so as to handle the node localization problem. The proposed work is implemented using MATLAB 8.2 whereas nodes are deployed in a random location within the desired network area. The parameters like computation time, percentage of localized node, and minimum localization error measures are utilized to analyse the potency of GWO rule with other variants of metaheuristics algorithms such as particle swarm optimization (PSO) and modified bat algorithm (MBA). The observed results convey that the GWO provides promising results compared to the PSO and MBA in terms of the quick convergence rate and success rate.

\section{Introduction}

In real-time environment, wireless sensor networks (WSNs) are deployed in a sensor field to screen the physical environment behaviors. From the most recent decades, the greater part of the scientists was pulled in extraordinary enthusiasm for WSN because of its minimal effort and low preparing capacities. WSNs have incomprehensible applications, for example, checking natural angles and physical marvels like temperature, environment observing, activity control observing, submerged acoustic observing, and patient social insurance checking. WSNs have many research issues that influence outline and execution of general system, for example, sending, time synchronization, restriction, least cost directing, and nature of administration and system security. Most of the articles and research proposals are introduced to solve these issues but still the challenging problem in WSN is localization [1]. With an example, in a general scenario if an unexpected nature disaster is occurred, sensor nodes are placed through air craft in stochastic manner. Those sensor nodes might not know their current locations and placing of GPS to all the sensor nodes is very expensive [2]. Sensor nodes with GPS, known as anchor nodes, transmit beacon messages to estimate the position of unknown nodes, which requires much power. The unknown node position is estimated using the distance between the anchor node (with known location) and unknown node (with unknown location). The overall performance of the network degrades if one node location is wrong; as a result, wrong data of anchor node area is proliferated.

In the past literature, wide range of localization algorithms and techniques are implemented to minimize the communication cost and to improve energy efficiency; however, a large portion of the calculations are application particular and the majority of the arrangements are not proper for extensive variety of WSNs. By and large, confinement is characterized into two unique classes, namely, extend based and run free restriction. Range measurement techniques help to estimate the location of sensor nodes in range based localization $[3,4]$. Range-free localization estimates the position of unknown nodes using the topology information and its connectivity with its adjacent nodes $[5,6]$. In existing 
location awareness approaches are commonly employed into two techniques; first one is based on distance or angle measurement and second one is hybridization of distance and angle. Received Signal Strength Indicator (RSSI) is most frequently used technique, where the node position is measured by the distance of nodes. Other location awareness approaches are as Time of Arrival (ToA), Angle-of-Arrival (AoA), Triangulation, and maximum Likelihood (ML).

Received Signal Strength Indicator (RSSI). The identification of unknown node is processed based on the attenuation of radio signal. However, the signal strength is high and the node is near to the sender and vice versa $[7,8]$.

Time of Arrival (ToA). TOA figures the separation by the variety of propagation time between two sensors with foreordained speed of signal proliferation $[9,10]$.

Angle-of-Arrival (AoA). Also called Direction of Arrival (DoA) methods ascertain the area by geometric directions with the point from where signals are obtained [11, 12].

Triangulation Method. Sensor positions are dictated by trigonometry laws of $\sin \varphi$ and $\cos \varphi$.

Maximum Likelihood (ML). ML ascertains the sensor position by diminishing the contrast between the computed distance and evaluated distance.

Many metaheuristics algorithms are applied to solve the localization issue in WSN, which drastically reduces the localization error. These algorithms belong to the family of trial and error problem solvers, which iteratively process the feasible solution and identify the nearest optimal solution to the various problems. In localization issues, various optimization algorithms like genetic algorithm, particle swarm optimization, shuffled frog leaping, cuckoo search, bat algorithms and so forth are aids to improvise the network performance by effective and efficient identification of unknown node position.

This paper is structured as follows: Section 2 discussed the literature survey of previous research in WSN localization. Section 3 presents a detailed review on grey wolf algorithm. Section 4 clarifies the localization issue moved toward utilizing the improvement strategies. Section 5 is about results and discussion based on the simulation work done and Section 6 presents conclusion and future direction for research path.

\section{Literature Survey}

In general, various localization procedures [13] are introduced to solve the localization problems in wireless sensor networks. These procedures are arranged in view of the three categories, namely, (a) gradient search technique, (b) nonhybrid optimization technique, and (c) hybrid optimization technique.

2.1. Gradient Search Technique. In gradient search techniques, identification of unknown node location is processed based on the first-order iterative methods. The following description explains the work carried out over past decades in solving localization issues. Firstly, DV-Hop Localization algorithm [14] utilizes the hop-based propagation model. In this algorithm distance of all nodes are shared with each other, whereas every unknown process a table to store the coordinates ( $x$ and $y$ ) of anchor nodes and the number of hop information from unknown node to the anchor node. Whenever neighbouring nodes provide the information of the unknown node, then the table is updated with the obtained positions; otherwise the table remains the same. The drawback of DV-Hop localization is whenever the size of network increases then the anchor nodes and unknown nodes are increased, respectively. This in turn returns the maximum cumulative error. This in turn provides the maximum localization error in finding unknown nodes. In addition, improved DV-Hop localization technique [15] is introduced to overcome the issues of DV-Hop algorithm. Mean correction factor of anchor nodes and average correction factor is computed in order to minimize the localization error. Later, Weighted DV-Hop localization algorithm is introduced with the slight modification in mean correction factor to obtain the mean hop distance error in the network and also to improve the accuracy of the unknown node position. In addition, weighted hyperbolic DV-Hop algorithm (WHDV-Hop) [16] presented with the combination of weighted DV-Hop and hyperbolic localization algorithms. WHDV-Hop algorithm requires a priori information of estimated position between the anchor nodes and node of interest and also requires the position of anchor nodes. This algorithm provides better accuracy with minimized mean square error and computational complexity.

Likewise, various techniques are presented to address the issues of localization problem. Some of them are as follows: Akyildiz et al. [17] and Boukerche et al. [18] provide a detailed survey of the significant literature. Niculescu and Nath [19] proposed an efficient localization system by extending the GPS capability with accurate positioning system (APS) to non-GPS nodes in ad hoc networks as anchors flow their location information to all nodes in the network. Bulusu et al. [20] introduced a refinement phase to improve the localization accuracy by measuring anchor distances from their adjacent nodes. Savvides et al. [21] addressed the issue of error accumulation which is solved with the aid of Kalman filter based on least square estimation [22] to concurrently discover the position of all sensor nodes.

Semidefinite programming depends on using convex optimization to address the node localization problem. Based on Biswas et al. [23] the slope seek strategy decides the utilization of an information examination procedure known as multidimensional scaling (MDS) in evaluating the position of obscure nodes. The calculation limits an individual fix by first figuring all match insightful most brief ways between sensors in the fix. At that point the MDS connected to these separations to get an underlying system. Positively, an outright guide is accomplished by utilizing the known node position. This methodology works well with some anchor and apparently high connectivity. 
2.2. Nonhybrid Optimization Technique. Even though gradient method solves the localization problem in WSN, it lags in solving large scale scenarios; in order to overcome this issue localization problem is considered as an optimization one. Nowadays optimization algorithm [24] plays a vital role in solving the issues in WSN. WSN is considered as the multimodal and multidimensional problem which diminishes the drawback by addressing the population based random techniques. Various optimization algorithms are so far introduced but only limited algorithms are used to address the issue of localization problem. Nonhybrid optimization algorithms are also termed as metaheuristics. Metaheuristic algorithms are very popular algorithms helping in finding the optimal solution in combinatorial problems. Some of the algorithms are more popular thus they are simulated annealing, genetic algorithm, particle swarm optimization algorithm, cuckoo search, bat algorithm and so on.

Simulated annealing by Kannan et al. [25] was the primary calculation used to detail the restriction as an improvement issue. When all is said and done, SA has a place with Monte Carlo technique to tackle the combinatorial advancement issue. It copies the conduct of physical tempering strategy for liquid materials. It starts with high temperature of a material and step by step cools into a base vitality crystalline structure. This algorithm categorizes into two phases: in first phase, the unknown node positions are estimated. In second phase, it utilizes the neighbourhood information to mitigate the flip ambiguity. Centralized infrastructure based network scenario is used to test the SAL method, where all the sensors transmit their information of estimated positions to a central station. This technique lags in error accumulation due to initial random position assignment.

Particle swarm optimization (PSO) is a very popular algorithm which mimics the behavior of birds flocking and fish schooling. PSO for node localization [26] is introduced to fine-tune the estimated position and to minimize the localization error. Author in that paper classified the process into two different process: first process the estimation of unknown node position is computed based on the improved version of DV-distance based method. In subordinate process, PSO algorithm is used to fine-tune the estimated position. In addition, various techniques with two neighbouring nodes and node selection priority are carried out to address the main issues of localization such as flip ambiguity, collective translation, and error propagation problem. Genetic based optimization mimics the behavior of biological evolution technique such as natural selection and genetic mechanism. In this work, author combined genetic algorithm with improved DV-Hop technique to enhance the localization of unknown nodes with respect to generic DV-Hop technique. This algorithm suffers in providing localization accuracy [27]. Cuckoo search algorithm based localization is introduced by Goyal and Patterh, [28]. With the help of CS author identified the unknown node position and then compared it with other metaheuristics methods.

2.3. Hybrid Optimization Technique. Hybrid optimization techniques determine that two or three metaheuristics algorithms are merged together to form a new optimization algorithm. These algorithms aid in efficient findings of optimal solution within the minimum computation time. Some of the researchers used the hybrid algorithm to solve the localization problem. Firstly, Niewiadomska-Szynkiewicz and Marks [29] proposed a hybrid algorithm with the combination of simulated annealing and genetic algorithm. This approach uses trilateration technique to improve the accuracy of unknown node position coordinates. At each generation, the computed objective value is measured if the obtained value is minimum the assumed threshold then stochastic selection is utilized to violate less proximity constraints. This technique lags in error accumulation due to gradual increase in trilateration. Modified bat algorithm based localization is presented by Goyal and Patterh, [30] which mimics the behavior of bats in finding prey and various kind of insects even at complete darkness. In general bats use ordinary sonar known as echolocation to distinguish prey and to dodge impediments. In this work, creator adjusted bat calculation with chemotactic development of bacterial scrounging calculation to enhance the restriction precision inside the short calculation time period.

To the best of our knowledge, so far, grey wolf optimization (GWO) algorithm was never used for localization problem. So, in this paper the grey wolf optimization algorithm is proposed to optimize the multimodal localization problem and it performs quite well in terms of identifying unknown node position and localization accuracy.

\section{Grey Wolf Optimization Algorithm}

Metaheuristic optimization algorithms are becoming more familiar in engineering applications because they (i) rely on rather easy concepts and being straightforward to implement; (ii) do not require gradient information; (iii) can bypass local optima; (iv) are often used in a wide range of issues covering different disciplines. Vast numbers of algorithms are introduced for different combinatorial optimization problems. Grey Wolf optimization is one of the new algorithms proposed by Mirjalili et al., [31] in 2016. This algorithm is inspired by the social behavior of grey wolves and it works on leadership hierarchy hunting strategy. Grey wolves are considered as the top-level predators; they live in a group size of 5-12 wolves. Based on the hunting strategy the grey wolves are classified into four categories such as alpha, beta, delta, and omega. Alpha wolves are leader of the bundle. This wolf has the authority to make decision for sleeping place, hunting, and so on. These wolves are otherwise called dominant wolves and they strictly instruct other wolves to follow his/her orders. An alpha wolf plays a major role in producing new solutions. Secondly, Beta wolves are second level of wolves next to the alpha wolves. These wolves are assistant wolves that guide the alpha wolves in decision-making. It also has certain rights to make decision whenever alpha wolves are passed away. These wolves listen to the alpha decision and provide response to the alpha. Thirdly, delta wolves are next level wolves which are also called subordinate wolves. These wolves are belonging to the categories of elders, sentinels, hunters, scouts, and caretakers. Deltas follow the instruction of alphas and betas and they manage next level wolves named 
omega. Finally, omega is the lowest ranking wolves and play the role of scapegoat. These wolves are must follow the instructions of all other dominant wolves. Omegas are not important wolves but, in some cases, they help others from facing internal problems.

GWO algorithm is one of the interesting algorithms due to the group hunting strategy. Based on Muro et al., grey wolf hunting is classified into three categories (i) tracking, chasing, and approaching the prey, (ii) pursuing, encircling, and harassing the prey until it stops moving, and (iii) attacking towards the prey. In GWO, symbolic representation of alpha, beta, and deltas is represented as $\alpha, \beta$, and $\delta$. Grey wolf optimization contributes in both the exploration and exploitation phase. Exploitation is to search optimal solution in a local search space. In grey wolf, encircling prey and attacking for prey are two exploitation phases used to explore the optimal solution in a local search space. Search for prey works as the exploration phase in which the grey wolves search for the prey in a global search space.

In encircling prey, grey wolves recognize the location of prey and encircle them. In this phase, the position vector of the prey is defined and other search agents adjust its position based on the best solution obtained. The equation of encircling prey is given below:

$$
\begin{aligned}
\vec{D} & =\left|\vec{C} \cdot \overrightarrow{X_{p}}(k)-\vec{X}(k)\right|, \\
\vec{X}(k+1) & =\overrightarrow{X_{p}}(k)-\vec{A} \cdot \vec{D},
\end{aligned}
$$

where $k$ represents the current iteration, $\vec{A}$ and $\vec{C}$ are coefficient vectors, position vector of the prey is represented as $\overrightarrow{X_{p}}, \vec{X}$ is the position vector, $\|$ is the absolute value, and . is an element-by-element multiplication.

The vectors $\vec{A}$ and $\vec{C}$ are computed as follows:

$$
\begin{aligned}
& \vec{A}=2 \vec{a} \cdot \vec{r}-\vec{a}, \\
& \vec{C}=2 \cdot \vec{r},
\end{aligned}
$$

where $\vec{a}$ is linearly decreased from 2 to 0 in each iteration and $\vec{r}$ is a random vector in $[0,1]$. The position of the search agent $[X, Y]$ is adjusted based on the position of the prey obtained so far $\left[X^{*}, Y^{*}\right]$. The coefficient vectors $\vec{A}$ and $\vec{C}$ are adjusted to achieve the best agent in different places.

In hunting phase, grey wolves are directed by alpha $(\alpha)$ and some contributions are also provided to beta $(\beta)$ and delta $(\delta)$. Initially, the best optimum is not identifiable due to vast search space whereas in the hunting strategy the alpha is considered as the first best candidate solution, beta is the second-best candidate solution and finally delta is the third best candidate solution. In all iterations, these three solutions are saved and updated to adjust the position of the lowest ranking solution omega. The equation of hunting strategy is formulated as follows:

$$
\begin{aligned}
& \vec{D}_{\alpha}=\left|\vec{C}_{1} * \vec{X}_{\alpha}-\vec{X}\right| \\
& \vec{D}_{\beta}=\left|\vec{C}_{2} * \vec{X}_{\beta}-\vec{X}\right|, \\
& \vec{D}_{\delta}=\left|\vec{C}_{3} * \vec{X}_{\delta}-\vec{X}\right|,
\end{aligned}
$$

where $\vec{D}_{\alpha}, \vec{D}_{\beta}$, and $\vec{D}_{\delta}$ are the modified distance vector between the alpha, beta, and delta position to the other wolves and $\vec{C}_{1}, \vec{C}_{2}$, and $\vec{C}_{3}$ are three coefficient vector aids in adjust distance vector and it is computed using (3). $\vec{X}$ position of vector of other grey wolf (omega).

$$
\begin{aligned}
& \vec{X}_{1}=\vec{X}_{\alpha}-\vec{A}_{1} *\left(\vec{D}_{\alpha}\right), \\
& \vec{X}_{2}=\vec{X}_{\beta}-\vec{A}_{2} *\left(\vec{D}_{\beta}\right), \\
& \vec{X}_{3}=\vec{X}_{\delta}-\vec{A}_{3} *\left(\vec{D}_{\delta}\right),
\end{aligned}
$$

where $\vec{X}_{1}$ is an obtained new position vector using alpha position $\vec{X}_{\alpha}$ and distance vector $\vec{D}_{\alpha}, \vec{X}_{2}$ denotes that new position vector obtained using beta position $\vec{X}_{\beta}$ and distance vector $\vec{D}_{\beta}, \vec{X}_{3}$ represents the new position vector computed using delta position $\vec{X}_{\delta}$ and distance vector $\vec{D}_{\delta}$, and $\vec{A}_{1}, \vec{A}_{2}$, and $\vec{A}_{3}$ are three coefficient vectors computed using (2).

$$
\vec{X}(k+1)=\frac{\sum_{i=1}^{n} \vec{X}_{i}}{n},
$$

where $\vec{X}(k+1)$ is new finalized new position vector computed by average sum of all positions obtained using alpha beta and delta wolf, and $n$ represent the three wolves' alpha, beta, and delta $(n=3)$.

Attacking prey phase helps candidate solution to identify the local solutions. In order to perform local search coefficient vector $\vec{A}$ fluctuates its range in the interval of $[-2 \mathrm{a}, 2 \mathrm{a}]$ whereas a is linearly decreasing its value from 2 to 0 over course of generations. If the value of coefficient vector $|\vec{A}|$ is lesser than 1 then search agents perform the local search. With these GWO operators, search agents update their positions using the location of alpha, beta, and delta and attack toward the prey. This operator intent to stagnate in local optimum to overcome this issue search for prey phase is introduced. This phase helps to diverge from each other to find for prey and converge to attack prey. If the value of coefficient vector $|\vec{A}|$ is greater than 1 then search agents diverges from the prey and finds the new prey. Likewise, the parameter $C$ vector is helpful in avoiding local optima, whereas $C$ vector value changes in a range of $[0,2]$.

The parameters $A$ and $C$ guide GWO algorithm to identify the optimum solutions in a global search space. Algorithm 1 shows the pseudocode to implement the GWO algorithm. Figure 1 shows the algorithmic flows of GWO.

\section{Formulation of WSN Localization Problem}

WSN node localization problem formulates using the single hop range based distribution technique to estimate the position of the unknown node coordinates $(X, Y)$ with the aid of anchor nodes (position of known nodes) coordinates $(x, y)$. Anchor nodes are provided with GPS device, so it has the capability of automatically determining its position. Most of the nodes in the WSN are not equipped with GPS due to high cost. To measure coordinates of $N$ unknown nodes, the procedure followed is given below. 


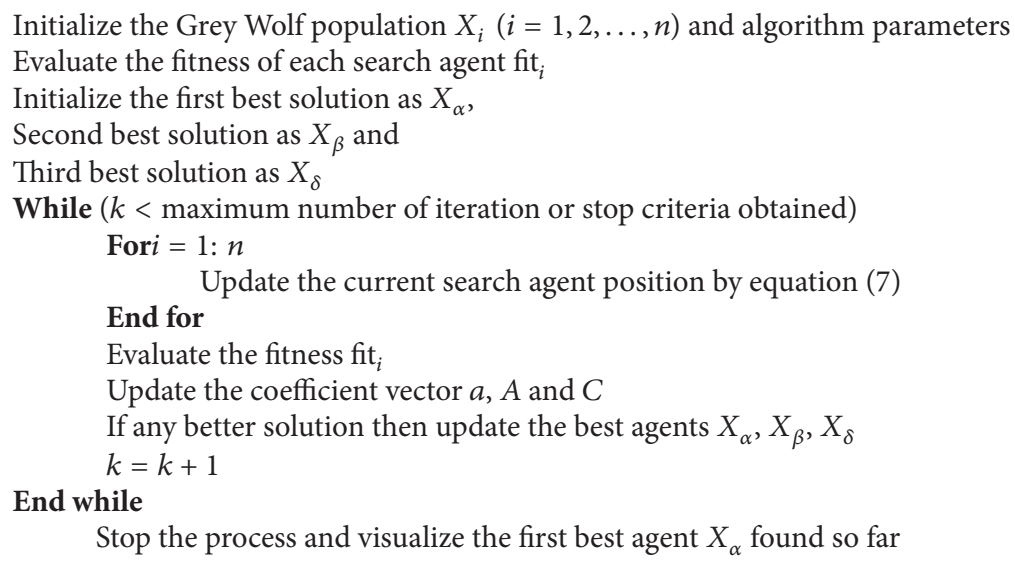

Algorithm 1: Pseudocode for the GWO [31].

Step 1. Randomly Initialize the $N$ unknown nodes and $M$ anchor nodes within the communication range $(R)$. Anchor nodes measure their position and communicate their coordinates to their neighbours. For all iterations, the node which settles at the end is termed as reference node and this node will act as anchor node.

Step 2. Three or more anchor nodes within the communication range of a node are considered as localized node.

Step 3. Neighbouring anchor node help to measure the location of localized node. Distance measurements are distracted due to environmental consideration; to eradicate it Gaussian noise $n_{i}$ is incorporated with the actual distance $d_{i}$.

$$
d_{i}=\sqrt{\left(X-x_{i}\right)^{2}+\left(Y-y_{i}\right)^{2}}
$$

The node estimates its distance from its anchor as $\widehat{d}_{i}=\left[d_{i}+\right.$ $\left.n_{i}\right]$.

$(X, Y)$ are the coordinates of unknown node/target node and $\left(x_{i}, y_{i}\right)$ are the coordinates of the $i$ th anchor node in the neighbourhood $[28,30]$.

Step 4. The optimization problem is formulated to minimize the error of localization problem. Each localizable target hub runs GWO calculation freely to restrict itself by identifying its position coordinates $(x, y)$. The target capacity of restriction issue is formulated as follows:

$$
f(x, y)=\min \left(\sum_{i=1}^{M}\left|d_{i}-\widehat{d}_{i}\right|\right),
$$

where $M$ is the number of anchor nodes within the transmission range $(R)$, of the target node.

Step 5. The localization error is characterized as the interval between the original and evaluated areas of an obscure node which is figured as the mean of square root of interval of evaluated node coordinates $\left(X_{i}, Y_{i}\right)$ and the original node arrangements $\left(e x_{i}, e y_{i}\right)$ for $i=1,2, \ldots$ (NL is the quantity of confined nodes) as demonstrated as follows:

$$
E_{L}=\frac{\sum_{i=M+1}^{N} \sqrt{\left(X_{i}-e x_{i}\right)^{2}+\left(Y_{i}-e y_{i}\right)^{2}}}{\left(N_{L}\right) * R} .
$$

Step 6. Repeat the Steps 2-5 until all unknown/target nodes get localized or no more nodes can be localized. Localization error $\left(E_{L}\right)$ and number of nonlocalized nodes $\left(N_{\mathrm{NL}}\right)$ aid to identify the performance of the localization algorithm. The number of nonlocalized nodes $\left(N_{\mathrm{NL}}\right)$ is identified based on the difference between the total number nodes and the number of nodes localized. The performance of the algorithm is better if it obtains minimum value of $N_{\mathrm{NL}}$ and $E_{L}$ [30].

In every evolution, the number of anchor nodes increases gradually based on the localized target nodes and these localized nodes are named referenced node. At the $k+1$ evolution these nodes will act as the anchor nodes and so on.

\section{Experimental Analysis}

In this segment, the point by point assessment of the GWO calculation is exhibited. For correlation, two different algorithms are utilized. They are altered PSO (PSO) [22] and another is MBA [20]. Since the GWO is a recently presented calculation with initiative chain of importance of chasing procedure, we are contrasting our GWO calculation with the other two algorithms. All the three calculations GWO, PSO, and MBA are executed in MATLAB 8.2 with Windows OS condition utilizing Intel Core i3, 3.30 GHz, $3 \mathrm{~GB}$ RAM. The populace size is introduced based on the quantity of sensor nodes deployed in the distribution range. The control parameter values for GWO are set according to the recommendations of the creators in [19]. The control parameter settings for the WSN and calculations are in Table 1.

5.1. Parameter Setup. In this paper, deployment area of wireless sensor network is considered as $300 \mathrm{~m} * 300 \mathrm{~m}$ with 300 


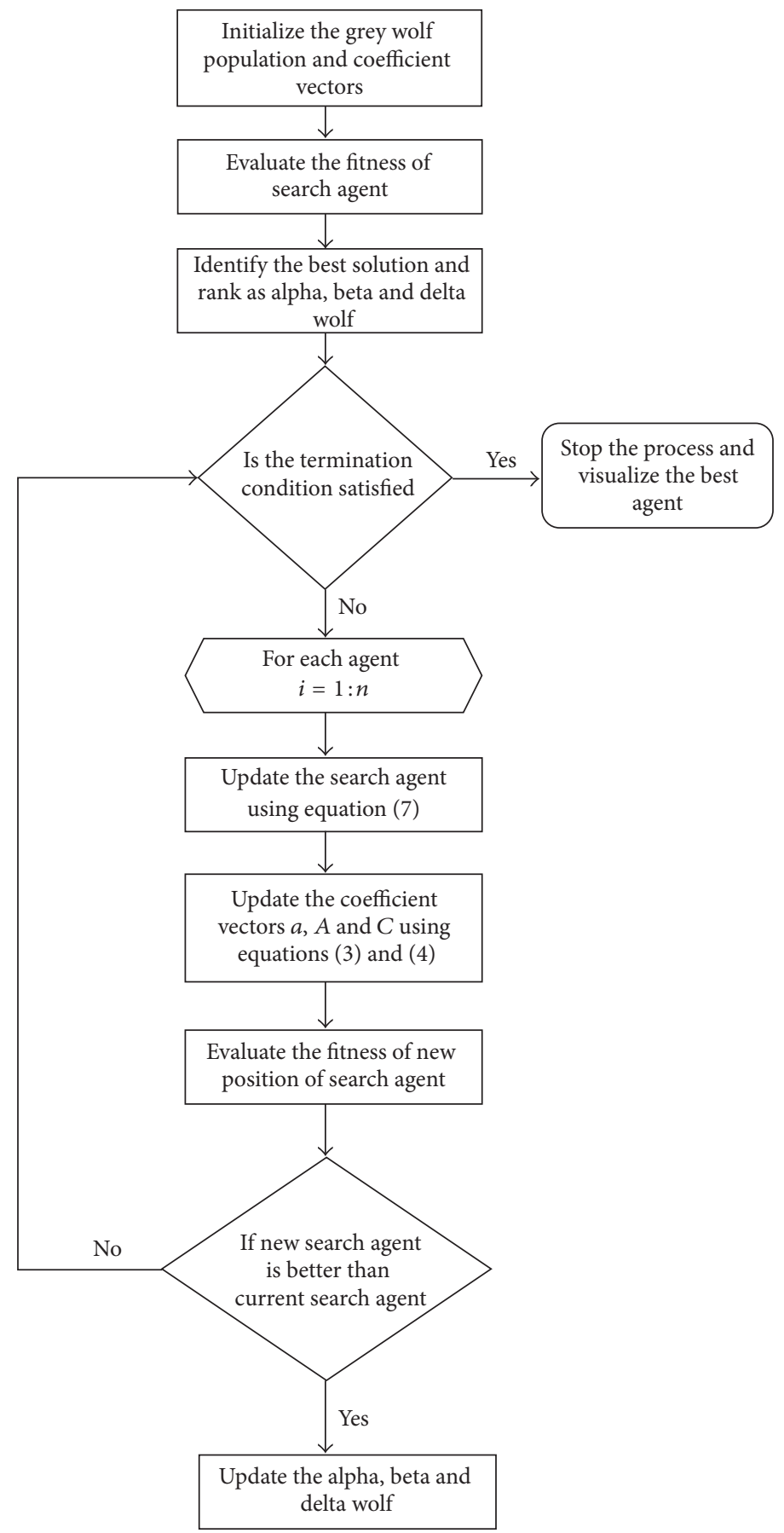

FIGURE 1: Flowchart of grey wolf optimization (GWO) algorithm.

sensor nodes. However, sensor nodes are scattered randomly in the simulation area, whereas the anchor nodes vary from $\sum_{i=1}^{10} i * 10$. For every optimization algorithm, the population size is fixed as 100 and the maximum number of iteration is 100. In PSO, social and cognitive parameter is assigned as $c 1=$ $c 2=2$, and momentum of inertia weight $\omega=1.2$ [22]. For MBA, the initial values for parameters pulse rate $(r)$ and loudness $(A)$ are assigned as 0.5 and $0.2 \mathrm{~ms}$, respectively [20].
For GWO, the parameter " $a$ " linearly decreases in the interval of [ 2 to 0 ] and the $C$ parameter linearly increases from 0 to 2.

5.2. Comparison of Results and Analysis of Localized Node. The performance of the GWO algorithm with other wellknown optimization algorithms such as PSO and MBA has been used to analyse the performance of the proposed work. 
TABLE 1: Parameter setting for WSN.

\begin{tabular}{lc}
\hline Sensor nodes & 300 \\
Anchor nodes & Varies on $\sum_{i=1}^{10} i * 10$ \\
Deployment area & $300 * 300 \mathrm{~m}^{2}$ \\
Transmission range (meters) & Varies on $\sum_{i=2}^{8} i * 5$ \\
Maximum number of iterations & 100 \\
\hline
\end{tabular}

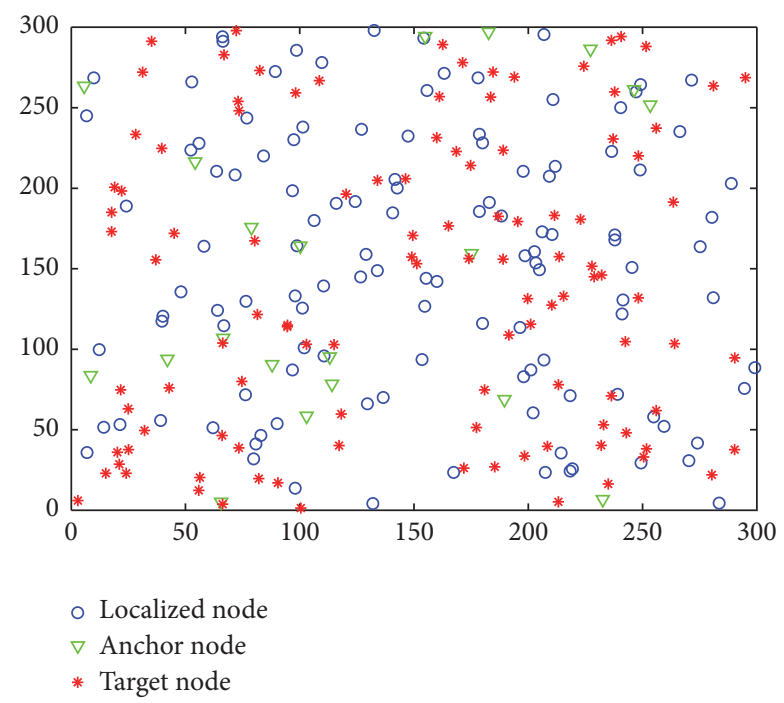

Figure 2: PSO for WSN localization.

Mean localization error (MLE), Computational time, and number of Localized nodes (NL) are considered to evaluate the performance of the GWO algorithm.

In general, 300 sensor nodes are placed randomly in a deployment area. Nodes are classified into three categories anchor (position known node), target (unknown position node), and localized (reference node or position identified so far) node. Figure 2 displays the findings of the localized node using the particle swarm optimization (PSO) algorithm. In generic PSO, the localized nodes are minimum due to its slow convergence rate. The anchor nodes are varied to test the efficiency of the algorithms. Figure 3 shows the graphical representation of node placement and the identification of unknown node positions with respect to the anchor nodes using modified bat algorithm. Figure 4 shows the localization of unknown nodes with the help of GWO and clearly it conveys that the number of nonlocalized nodes is minimum.

The results are analysed using the parameters such as mean localization error (MLE), Computational time, and number of Localized nodes (NL). The anchor nodes are varied from 10 to 100 for better efficiency in identifying unknown node positions. Initially, sensor nodes are scattered with 10 anchor nodes in the deployment area. PSO algorithm is applied into that scenario to identify the unknown node position. The results of the PSO with 10 anchor nodes are measured in Table 2. Likewise, MBA is used to identify the unknown node position whereas it localizes 178 nodes in deployed 300 sensor nodes. When the number of anchor nodes increases then the localized nodes are identical; the

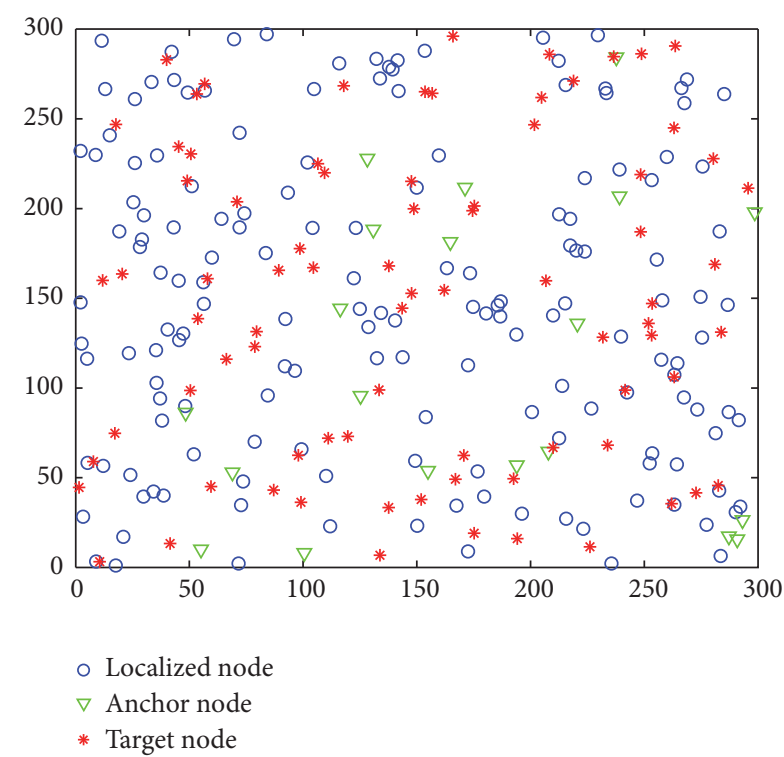

FIgURE 3: MBA for WSN localization.

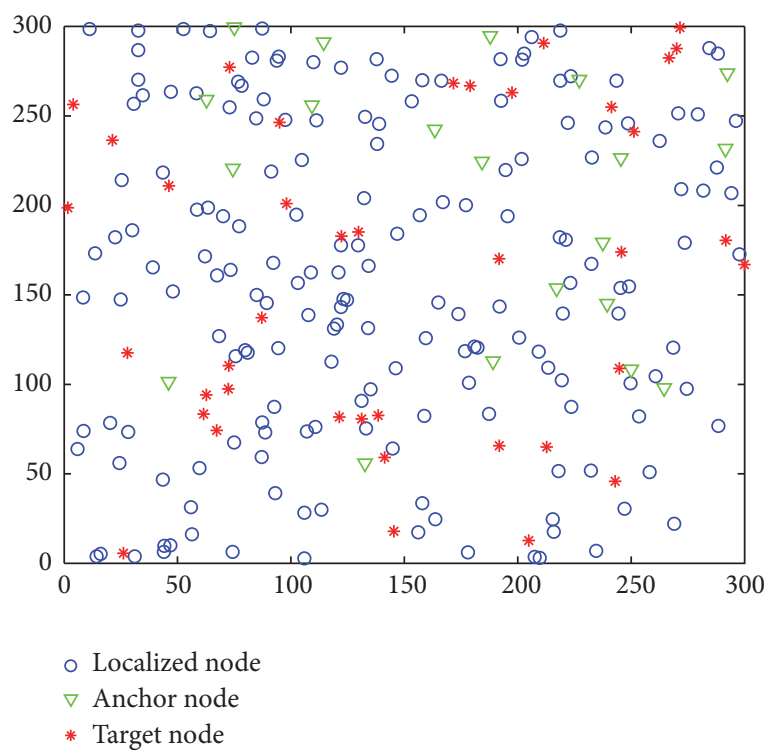

FIGURE 4: GWO for WSN localization.

performance has not been improved. In this work, GWO performs better in terms of identifying maximum number of unknown node positions. The leadership hierarchy and hunting strategy are the backbone of the GWO. This strategy helps a lot to identify the unknown node. The parameter " $a$ " gradually decreases to enhance the performance of in terms of local (unknown which is nearest to the anchor node) and global search (unknown node which is far away from the anchor). The performance of the GWO algorithm improved drastically and those results are represented in Table 2. Figure 5 provides pictorial representation of the proposed algorithm performance with other metaheuristics in terms of the minimum localization error. 
TABLE 2: Comparative analysis of mean localization error (MLE), computation time, and number of localized node (NL).

\begin{tabular}{lccccccccc}
\hline \multirow{2}{*}{ Anchor } & \multicolumn{3}{c}{ PSO } & \multicolumn{3}{c}{ MBA } & \multicolumn{3}{c}{ GWO } \\
& MLE (\%) & Time (s) & NL & MLE (\%) & Time (s) & NL & MLE (\%) & Time (s) & NL \\
\hline 10 & 0.5559 & 4.17 & 132 & 0.6982 & 3.99 & 178 & 0.7771 & 1.98 & 185 \\
20 & 0.5488 & 3.61 & 139 & 0.6422 & 2.48 & 186 & 0.7762 & 2.21 \\
30 & 0.5357 & 3.03 & 142 & 0.6358 & 3.25 & 195 & 0.7758 & 2.60 & 213 \\
40 & 0.4787 & 5.11 & 154 & 0.6169 & 2.47 & 205 & 0.7511 & 2.50 & 221 \\
50 & 0.4594 & 4.59 & 161 & 0.5798 & 2.25 & 210 & 0.7043 & 2.37 \\
60 & 0.4592 & 5.59 & 173 & 0.5747 & 3.33 & 240 & 0.7011 & 2.55 \\
70 & 0.4445 & 5.01 & 182 & 0.5425 & 1.86 & 246 & 0.6983 & 249 \\
80 & 0.4433 & 5.58 & 196 & 0.5369 & 4.13 & 251 & 0.6974 & 2.06 \\
90 & 0.4247 & 5.22 & 208 & 0.5205 & 1.75 & 258 & 0.6779 & 262 \\
100 & 0.3054 & 5.93 & 219 & 0.5021 & 2.55 & 260 & 0.6586 & 2.51 & 279 \\
\hline
\end{tabular}

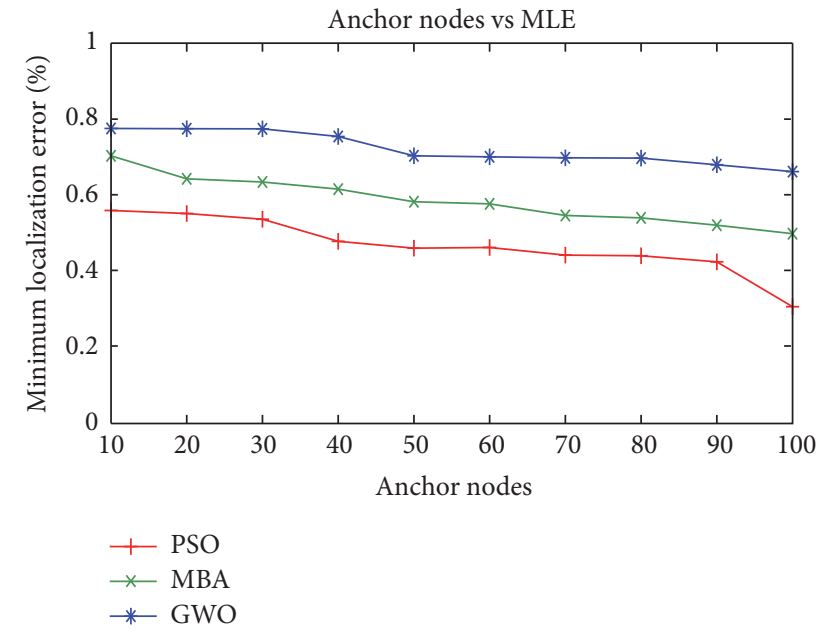

FIGURE 5: Comparison results of anchor node versus MLE.

At the same time, we have measured running time of each algorithm with respect to increase in number of anchor nodes. All the computations are performed in the same system. The running time is measured in terms of seconds over 100 iterations. Figure 6 display the running time of the all the algorithms. From that we understand that the GWO algorithm finds the maximum number of unknown node positions within the minimum computation time. Finally, the count of localized nodes is measured in Table 2 and it is plotted in Figure 7. GWO algorithm converges very fast and achieves the optimal result by identifying maximum number of localized nodes. From the observed results, minimum mean localization error (MLE) is attained when compared to the other algorithms as shown in Figure 7. GWO provides better localization accuracy than MBA and PSO algorithm.

5.3. Localization Results under Different Transmission Range. Transmission range of sensor node is considered as another

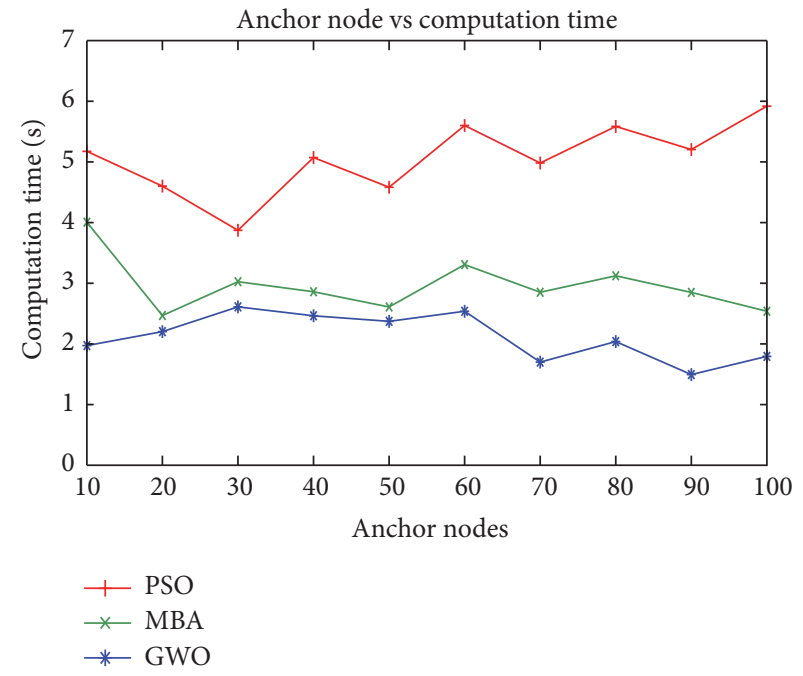

FIGURE 6: Comparison results of anchor node versus computation Time.

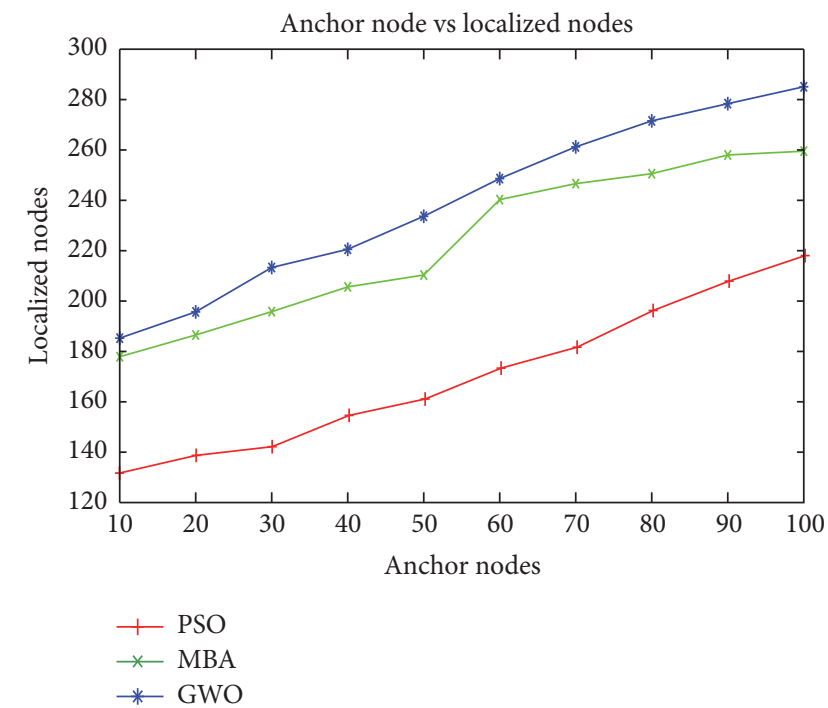

FIGURE 7: Comparison results of anchor node versus localized Node. 
TABLE 3: Result by varying number of transmission range.

\begin{tabular}{lccc}
\hline \multirow{2}{*}{ Transmission range (meters) } & \multicolumn{3}{c}{ Localized nodes } \\
& PSO & MBA & GWO \\
\hline 10 & 86 & 118 & 125 \\
15 & 101 & 136 & 148 \\
20 & 110 & 149 & 156 \\
25 & 128 & 167 & 173 \\
30 & 133 & 175 & 184 \\
35 & 140 & 180 & 193 \\
40 & 149 & 191 & 209 \\
\hline
\end{tabular}

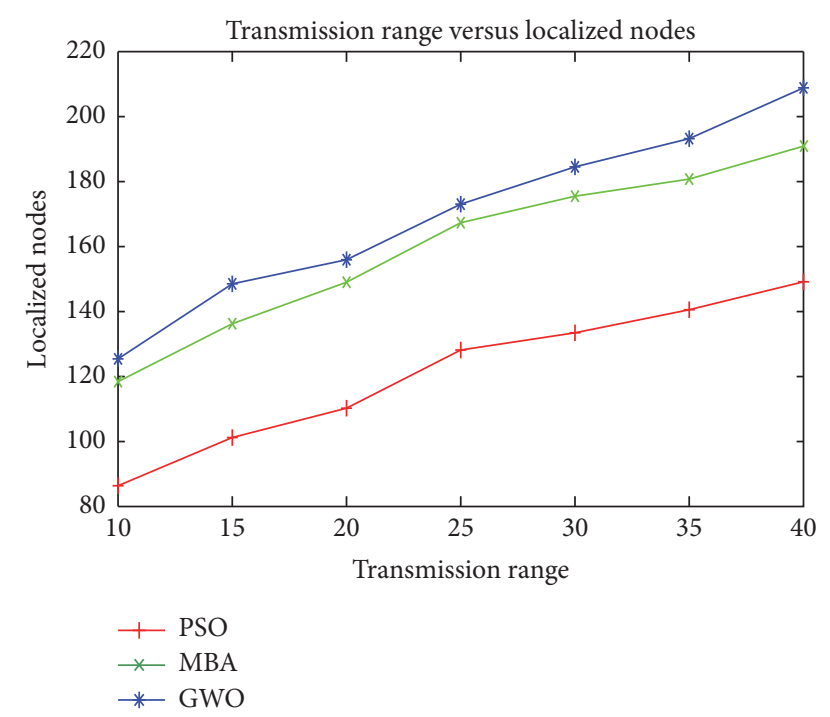

Figure 8: Comparison by varying Transmission range and obtained localized node.

parameter in wireless sensor network localization. The number of localized node increases gradually when the transmission range of sensor nodes increases which in turn obtains the minimum localization error. The transmission range of sensor nodes starts from 10 meters and gradually increases with 5 meters to analyse the performance of the algorithm. When the transmission range of the sensor node increases, the proposed algorithm obtains better result in terms of location accuracy. Table 3 shows the measured value of localized nodes with respect to the variation in transmission ranges. In general, GWO has three best solutions such as alpha, beta, and delta that are mapped as first neighbour, second neighbour, and third neighbour anchor node. This algorithm works as similar as triangulation method, but it enhances the existing method by obtaining better localization accuracy in both small and large scale environment. Figure 8 clearly shows that when the range of sensor nodes increases then the number of localized nodes increases and reduces the localization error. GWO algorithm provides the best performance compared to all other metaheuristic algorithms.

5.4. Observations. The proposed GWO algorithm for node localization problem is successfully implemented and the observed results show that the grey wolf optimization algorithm estimates the unknown node location and provides minimum localization error compared to other metaheuristic algorithms PSO and MBA. GWO algorithm is better due to its hierarchical leadership strategy. This strategy improves the solution (unknown node position) with the aid of three known solutions. It outperforms the triangulation methods for large scale environment.

Finally, this paper concludes the results that the GWO algorithm provides better performance in terms of location accuracy and minimization of localization error. Faster convergence rate and estimating the location of the node within the minimum computation time are additional advantage of GWO.

\section{Conclusion}

This review exhibited another swarm-based advancement calculation motivated by the leadership hierarchy of chasing conduct of dark wolves. This GWO encased three leaders to recreate the scan for prey, enclosing prey, and initiative chase conduct of dark wolves. GWO was identified to be sufficient competitive with other state-of-the-art metaheuristic methods to analyse exploration, exploitation, nearby optima evasion, and convergence behavior. Grey wolf optimization algorithm was never used for localization problem. This paper utilized GWO algorithm for localization problem and provided better results in finding location of unknown nodes. The numerical computation results such as convergence rate (minimum computation time) and success rate (maximum number of localized nodes) of proposed GWO algorithms are noted and it is compared with other variants such as PSO and MBA algorithms. The results of GWO algorithm are compared with other metaheuristics approaches and thereby it achieves better performance with respect to maximum number of localized nodes. Further, this algorithm can be tested in movable node networks such as Mobile ad hoc networks (MANET). In future, GWO algorithm can integrate with other variants of metaheuristic algorithms to form a hybrid algorithm for efficient move in convergence and diversity over the identification of maximum number of unknown node positions.

\section{Conflicts of Interest}

The authors declare that there are no conflicts of interest regarding the publication of this paper.

\section{References}

[1] N. A. A. Alrajeh, M. Bashir, and B. Shams, "Localization techniques in wireless sensor networks," International Journal of Distributed Sensor Networks, vol. 9, no. 6, pp. 1-9, 2013.

[2] D. Lavanya and S. K. Udgata, "Swarm intelligence based localization in wireless sensor networks," in Multi-Disciplinary Trends in Artificial Intelligence, vol. 7080, pp. 317-328, Springer, 2011. 
[3] K. K. Almuzaini and A. Gulliver, "Range-based localization in wireless networks using density-based outlier detection," Wireless Sensor Network, vol. 2, pp. 807-814, 2010.

[4] Y. Zhang, W. Wu, and Y. Chen, "A range-based localization algorithm for wireless sensor networks," Journal of Communications and Networks, vol. 7, no. 4, pp. 429-437, 2005.

[5] R. Stoleru, T. He, and J. A. Stankovic, "Range-free localization," Advances in Information Security, vol. 30, pp. 3-31, 2007.

[6] C.-C. Chen, C.-Y. Chang, and Y.-N. Li, "Range-free localization scheme in wireless sensor networks based on bilateration," International Journal of Distributed Sensor Networks, vol. 2013, Article ID 620248, 10 pages, 2013.

[7] J. Hightower, G. Boriello, and R. Want, "SpotON: an indoor 3D location sensing technology based on RF signal strength," Tech. Rep. 2000-02-02, University of Washington, 2002.

[8] Y.-C. Tseng, S.-P. Kuo, H.-W. Lee, and C.-F. Huang, "Location tracking in a wireless sensor network by mobile agents and its data fusion strategies," in Proceedings of the International Workshop on Information Processing in Sensor Networks (IPSN '03), Palo Alto, Calif, USA, April 2003.

[9] A. Harter, A. Hopper, P. Steggles, A. Ward, and P. Webster, "The anatomy of a context-aware application," in Proceedings of the 5th Annual ACM/IEEE International Conference on Mobile Computing and Networking (MOBICOM '99), Seattle, Wash, USA, August 1999.

[10] N. B. Priyanath, A. Chakraborty, and H. Balakrishna, "The Cricket location-support system," in Proceedings of the 6th Annual International Conference on Mobile Computing and Networking (MobiCom '00), Boston, Mass, USA, August 2000.

[11] A. Nasipuri and K. Li, "A directionality based location discovery scheme for wireless sensor networks," in Proceedings of the 1st ACM International Workshop on Wireless Sensor Networks and Applications (WSNA '02), pp. 105-111, ACM, Atlanta, Ga, USA, September 2002.

[12] D. Niculescu and B. Nath, "Ad Hoc Positioning System (APS) using AoA," in Proceedings of the IEEE Conference on Computer Communications (INFOCOM '03), San Francisco, Calif, USA, 2003.

[13] G. Mao, B. Fidan, and B. D. O. Anderson, "Wireless sensor network localization techniques," Computer Networks, vol. 51, no. 10, pp. 2529-2553, 2007.

[14] J. F. Kurose and K. W. Ross, Computer Networking: A-Top Down Approach Featuring the Internet, Longman, Reading, Mass, USA, 2013.

[15] S. Mingyu and Z. Yin, "DV-hop localization algorithm based on improved average hop distance and estimate of distance," Application Research of Computers, vol. 28, no. 2, pp. 648-650, 2011.

[16] J. Mass-Sanchez, E. Ruiz-Ibarra, J. Cortez-González, A. Espinoza-Ruiz, and L. A. Castro, "Weighted hyperbolic DVhop positioning node localization algorithm in WSNs," Wireless Personal Communications, 2016.

[17] I. F. Akyildiz, W. Su, Y. Sankarasubramaniam, and E. Cayirci, "Wireless sensor networks: a survey," Computer Networks, vol. 38, no. 4, pp. 393-422, 2002.

[18] A. Boukerche, H. A. B. F. Oliveira, E. F. Nakamura, and A. A. F. Loureiro, "Localization systems for wireless sensor networks," IEEE Wireless Communications, vol. 14, no. 6, pp. 6-12, 2007.

[19] D. Niculescu and B. Nath, "Ad hoc positioning system (APS)," in Proceedings of the IEEE Global Telecommunications Conference
(GLOBECOM '01), vol. 5, pp. 2926-2931, IEEE, San Antonio, Tex, USA, November 2001.

[20] N. Bulusu, D. Estrin, L. Girod, and J. Heidemann, "Scalable coordination for wireless sensor networks: self-configuring localization systems," in Proceedings of the 6th International Symposium on Communication Theory and Applications (ISCTA '01), Ambleside, UK, July 2001.

[21] A. Savvides, H. Park, and M. B. Srivastava, "The bits and flops of the n-hop multilateration primitive for node localization problems," in Proceedings of the 1st ACM International Workshop on Wireless Sensor Networks and Applications (WSNA '02), pp. 112-121, ACM, September 2002.

[22] M. Di Rocco and F. Pascucci, "Sensor network localisation using distributed extended Kalman filter," in Proceedings of the IEEE/ASME International Conference on Advanced Intelligent Mechatronics (AIM '07), pp. 1-6, IEEE, September 2007.

[23] P. Biswas, T.-C. Lian, T.-C. Wang, and Y. Ye, "Semidefinite programming based algorithms for sensor network localization," ACM Transactions on Sensor Networks, vol. 2, no. 2, pp. 188220, 2006.

[24] S. Yun, J. Lee, W. Chung, E. Kim, and S. Kim, "A soft computing approach to localization in wireless sensor networks," Expert Systems with Applications, vol. 36, no. 4, pp. 7552-7561, 2009.

[25] A. Kannan, G. Mao, and B. Vucetic, "Simulated annealing based localization in wireless sensor network," in Proceedings of the 30th IEEE Conference on Local Computer Networks, pp. 513-514, Sydney, Australia, November 2011.

[26] P. H. Namin and M. A. Tinati, "Node localization using particle swarm optimization," in Proceedings of the 7th International Conference on Intelligent Sensors, Sensor Networks and Information Processing (ISSNIP '11), pp. 288-293, IEEE, Adelaide, Australia, December 2011.

[27] B. Peng and L. Li, "An improved localization algorithm based on genetic algorithm in wireless sensor networks," Cognitive Neurodynamics, vol. 9, no. 2, pp. 249-256, 2015.

[28] S. Goyal and M. S. Patterh, "Wireless sensor network localization based on cuckoo search algorithm," Wireless Personal Communications, vol. 79, no. 1, pp. 223-234, 2014.

[29] E. Niewiadomska-Szynkiewicz and M. Marks, "Optimization schemes for wireless sensor network localization," The International Journal of Applied Mathematics and Computer Science, vol. 19, no. 2, pp. 291-302, 2009.

[30] S. Goyal and M. S. Patterh, "Modified bat algorithm for localization of wireless sensor network," Wireless Personal Communications, vol. 86, pp. 657-670, 2015.

[31] S. Mirjalili, S. M. Mirjalili, and A. Lewis, "Grey wolf optimizer," Advances in Engineering Software, vol. 69, pp. 46-61, 2014. 


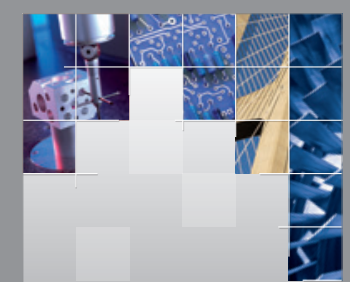

\section{Enfincering}
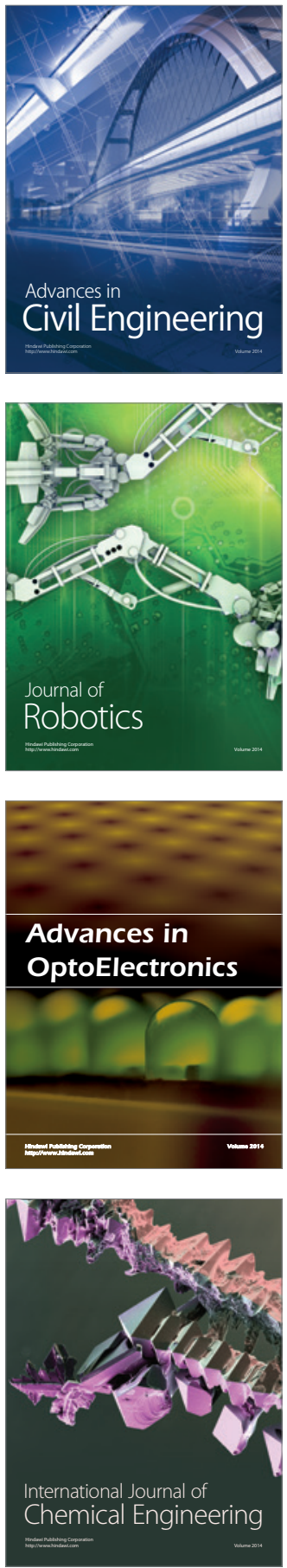

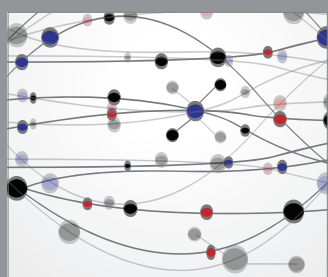

The Scientific World Journal

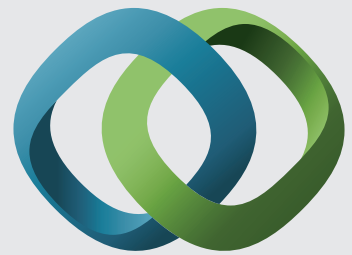

\section{Hindawi}

Submit your manuscripts at

https://www.hindawi.com
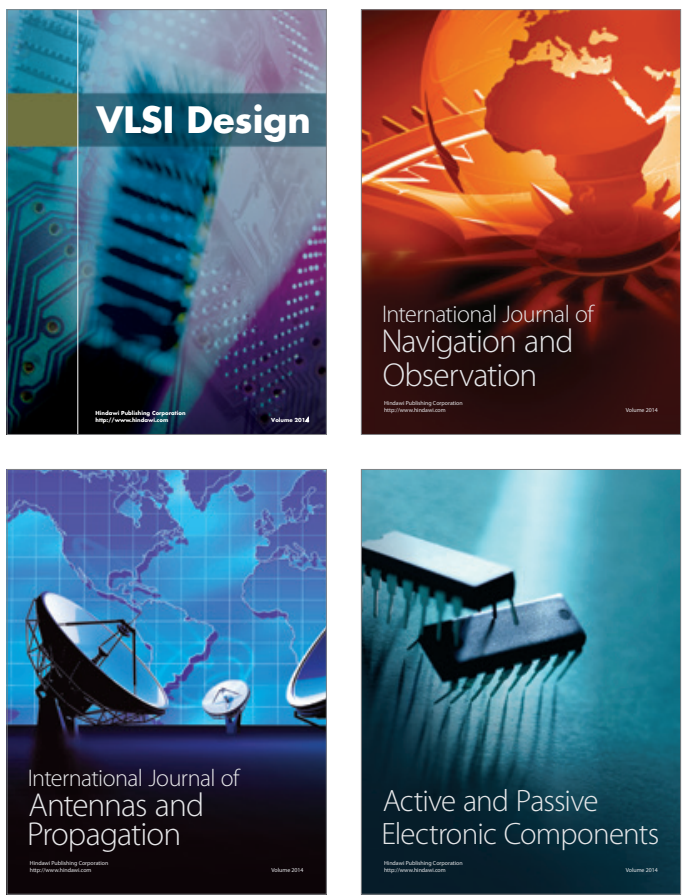
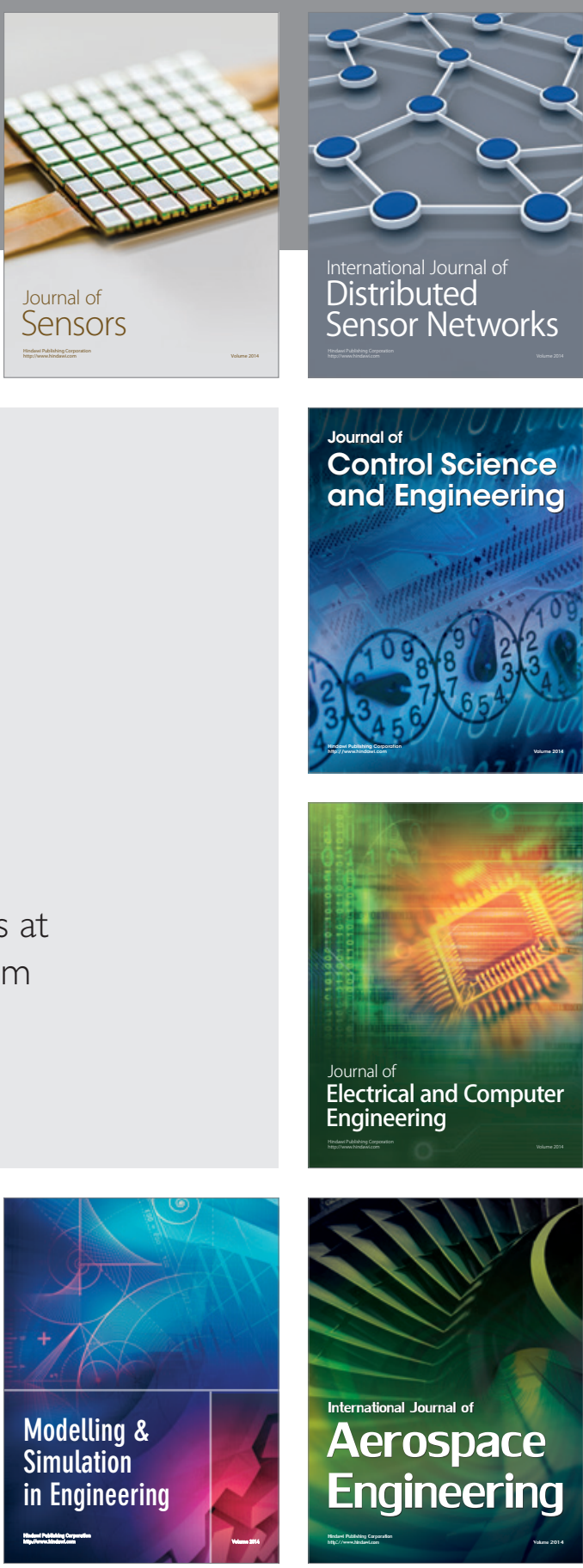

International Journal of

Distributed

Sensor Networks

$-$

Joumal of

Control Science

and Engineering
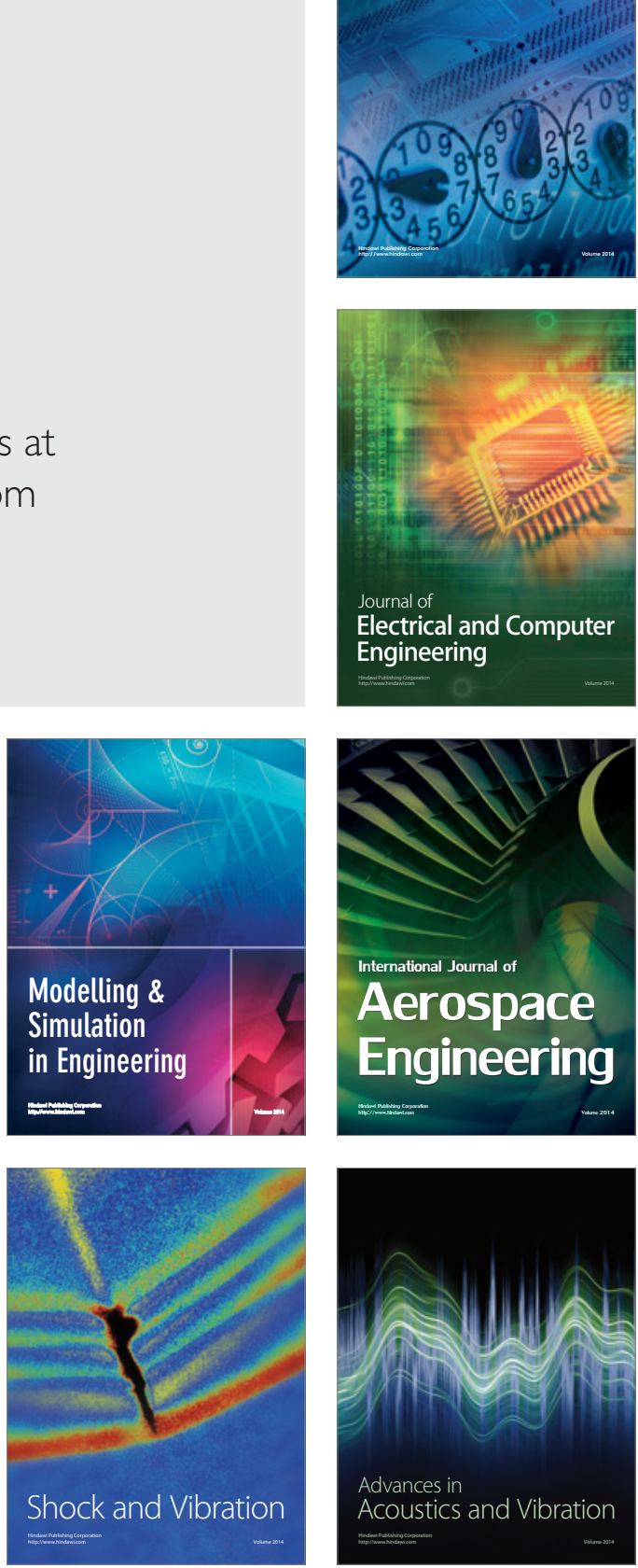